\title{
Distribuição espacial e qualidade dos cursos de graduação em fisioterapia no Brasil
}

\author{
Space distribution and quality of graduation courses in \\ physiotherapy in Brazil
}

\author{
Erica Silva de Souza Matsumura', Alcinês da Silva Sousa Júnior², \\ Nelson Veiga Gonçalves ${ }^{3}$, Katia Simone Kietzer ${ }^{4}$, Leny Silene de Freitas Castro ${ }^{5}$ \\ 'Laboratório de Epidemiologia e Geoprocessamento da Amazônia, Programa de Pós-graduação em Ensino em \\ Saúde na Amazônia, Departamento Saúde Comunitária, Universidade do Estado do Pará, Belém (PA), Brasil. \\ ${ }^{2}$ Laboratório de Epidemiologia e Geoprocessamento da Amazônia, Programa de Pós-graduação em Saúde, \\ Ambiente e Sociedade na Amazônia, Universidade do Estado do Pará, Belém (PA), Brasil. \\ ${ }^{3}$ Laboratório de Epidemiologia e Geoprocessamento da Amazônia, Programa de Pós-graduação em Saúde \\ Pública e de Medicina Tropical de Lisboa, Departamento Saúde Comunitária, Universidade do Estado do Pará, \\ Belém (PA), Brasil. \\ ${ }^{4}$ Programa de Pós-graduação em Ensino em Saúde na Amazônia, Departamento de Ciências Morfológicas e \\ Fisiológicas, Universidade do Estado do Pará, Belém (PA), Brasil. \\ ${ }^{5}$ Programa de Pós-graduação em Doenças Tropicais, Departamento de Ciências do Movimento Humano, \\ Universidade do Estado do Pará, Belém (PA), Brasil.
}

Recebido. Ago. 28, 2018 Aceito: Mar. 5, 2019

\section{COMO CITAR ESTE ARTIGO}

Matsumura ESS, Sousa Júnior AS,

Gonçalves NV, Kietzer KS, Castro LSF.

Distribuição espacial e qualidade dos cursos de graduação em fisioterapia no Brasil. Interdisciplinary Journal of Health Education. 2020Jan-Jun;5(1):12-21. https://doi.org/10.4322/ijhe.2020.002

\section{CORRESPONDÊNCIA}

Erica Silva de Souza Matsumura Centro de Ciencias Biológicas e da Saúde, Universidade do Estado do Pará Travessa Perebebuí, 2623 CEP 66095-015, Belém (PA), Brasil Tel.: (91) 98895-0189 Tel.: (91) 98895-0189

\section{FONTE DE FINANCIAMENTO}

Recursos próprios.

\section{CONFLITO DE INTERESSE}

Os autores declararam não

haver conflitos de interesse.

O estudo foi realizado na Universidade do Estado do Pará, Belém (PA), Brasil.

Este trabalho é parte da dissertação mestrado do Programa de

Pós-Graduação em Ensino em

Saúde na Amazônia - Belém /PA de

Erica Silva de Souza Matsumura.

Todos os autores leram e aprovam a versão final submetida ao

Interdisciplinary Journal of Health Education (IJHE).

\section{RESUMO}

Objetivo: Buscou-se compreender a distribuição espacial dos cursos de fisioterapia no Brasil relacionando com seus indicadores educacionais que avaliam a qualidade. Método: Foi realizado um estudo quantitativo, analítico e de caráter descritivo a partir de análise dos dados secundários coletados no Portal do Ministério da Educação, no Instituto Nacional de Estudos e Pesquisas, referentes às instituições de educação superior e cursos cadastrados autorizados para os cursos de graduação em fisioterapia através do portal eletrônico e-MEC, via busca interativa. Resultado: Foram identificadas 632 IES com o curso de fisioterapia na modalidade de ensino presencial, com concentração e maioria dos cursos que apresentaram Conceito Preliminar de Curso localizados na região Sudeste. Conclusão: O presente estudo permitiu caracterizar a distribuição e a qualidade dos cursos de graduação em fisioterapia no Brasil por regiões e estados.

PALAVRAS-Chave: Ensino superior. Avaliação. Formação profissional. Educação em saúde. Fisioterapia.

\section{ABSTRACT}

Objective: We sought to understand the spatial distribution of physiotherapy courses in Brazil, relating to their educational indicators that assess quality. Method: A quantitative, analytical and descriptive study was carried out based on the analysis of secondary data collected on the Ministry of Education Portal, at the National Institute of Studies and Research, referring to institutions of higher education and registered courses authorized for courses of graduation in physiotherapy through the electronic portal e-MEC, via interactive search. Result: 632 Higher Education Institutions were identified with the physiotherapy course in the face-to-face teaching modality, with concentration and most of the courses that presented a Preliminary Course Concept located in the Southeast region. Conclusion: The present study allowed to characterize the distribution and quality of undergraduate courses in physiotherapy in Brazil by regions and states.

KEYWORDS: Higher education. Evaluation. Professional qualification. Health education. Physiotherapy. 


\section{Introdução}

Após as duas Guerras Mundiais milhões de feridos e lesados contribuíram para o aumento da necessidade pelos serviços de Fisioterapia, principalmente nos Estados Unidos. Outro fator que contribuiu, em grande parte para o desenvolvimento da Fisioterapia, foram as epidemias de poliomielite ${ }^{1}$.

A evolução da formação em fisioterapia no Brasil ocorreu de forma gradual entre as décadas de 1970 e 1980. Essa evolução sofreu influências dos contextos políticos, econômicos e sociais desde a sua regulamentação que aconteceu em 1969, quando existia apenas seis cursos de graduação no país. Transcorridos 15 anos surgiram mais 16 cursos totalizando 22 cursos em 1984. Até o ano de 1995 havia um total de 63 cursos$^{2}$ acelerando o crescimento da abertura de cursos a partir de $1997^{3}$

Esse crescimento ocorreu com a aprovação da Lei as Diretrizes e Bases da Educação Nacional (LDB), no ano de 1996, culminando uma política de incentivos ao crescimento da educação superior, gerando um processo acelerado de expansão do ensino, principalmente no setor privado com a autorização pelo MEC para a abertura de novos cursos.

ApósLDBeoPlano Nacional daEducação(PNE) paraodecênio2001-2010contribuíram para o aumento da oferta e do crescimento do sistema educacional no país, favorecendo a abertura indiscriminada de cursos, sem o estudo das demandas específicas de cada região, gerando desequilíbrio na distribuição regional, além da privatização das IES com padrões mínimos de qualidade ${ }^{4}$.

O sistema de ensino superior cresceu no quantitativo de instituições e alunos, mas não refletiu na qualidade e na regularidade dos cursos, ocorrendo baixa frequência, baixa avaliação do aprendizado e baixa qualidade de ensino ${ }^{5}$.

Foi neste cenário que os cursos de Fisioterapia se inseriram. Houve uma ampliação no número de cursos e vagas, desencadeando os mesmos problemas que os demais cursos de graduação no Brasil, destacando-se a concentração geográfica e a privatização do ensino ${ }^{2}$. Essa concentração geográfica é relevante e ao mesmo tempo preocupante, pois reflete na assistência à população onde há claramente a carência de profissionais em várias regiões².

Neste contexto, o presente estudo buscou compreender a distribuição espacial dos cursos de fisioterapia no Brasil relacionando com seus indicadores educacionais que avaliam a qualidade, com o objetivo de disponibilizar informações que fundamentem a melhor distribuição de cursos novos e o planejamento e implementação de políticas de qualificação para os cursos de Fisioterapia.

\section{Regionalização e contextualização socioeconômica dos cursos de fisioterapia}

A regionalização se destaca como uma ferramenta de atuação do Estado, abrangendo recortes espaciais para fins de planejamento e gestão territorial de políticas públicas. Utilizando de diversos critérios para identificação e desenhos de cada região, em função dos objetivos específicos e dos diversos contextos socioeconômicos específicos ${ }^{6}$. $\mathrm{Na}$ área da saúde, a regionalização tem o objetivo de promover o uso mais eficiente dos recursos no território, ampliar o acesso e a qualidade da atenção à saúde ${ }^{7}$.

Um dos principais problemas no setor da saúde no mundo está na desigualdade da distribuição de profissionais da área da saúde entre as áreas rurais e urbanas, e capitais e interiores. A Organização Mundial da Saúde (OMS) ${ }^{8}$ recomenda políticas de recrutamento e fixação de profissionais da área médica para facilitar o acesso à saúde para essas populações.

Entre as diversas limitações da educação superior no Brasil, os principais problemas ressaltados foram a falta de planejamento, a baixa qualidade do ensino e o desequilíbrio entre as áreas de conhecimento e a regionalização? .

A desigualdade na distribuição dos cursos da área da saúde é um fator relevante e ao mesmo tempo preocupante, pois reflete na assistência à população, pela carência de 
profissionais em determinada região, enquanto há concentração maior de profissionais em outras regiões ${ }^{2}$.

\section{Indicadores educacionais de qualidade do Ensino Superior}

O Sistema Nacional de Avaliação da Educação Superior (SINAES), criado pela Lei $\mathrm{n}^{\circ}$ 10.861, de14 de abril de 2004, segue as diretrizes da Comissão Nacional de Avaliação da Educação Superior (CONAES), e o responsável pela organização de todo o processo avaliativo fica a cargo do Instituto Nacional de Estudos e Pesquisas Educacionais Anísio Teixeira ${ }^{10}$.

O SINAES é o responsável por avaliar a graduação, atribuindo "conceitos", que direcionam para a melhoria da qualidade da educação superior e da formação acadêmica. Suas avaliações analisam a qualidade das IES, dos cursos de graduação e do desempenho dos estudantes ${ }^{10}$.

A avaliação do desempenho dos estudantes dos cursos de graduação é realizada por meio do Exame Nacional de Desempenho de Estudantes (ENADE), que objetiva avaliar o desempenho dos estudantes, suas habilidades e competências. De acordo com a definição do $\operatorname{INEP}^{10}$ (p. 38):

O ENADE avalia o rendimento dos concluintes dos cursos de graduação, em relação aos conteúdos programáticos, habilidades e competências adquiridas em sua formação. O exame é obrigatório e a situação de regularidade do estudante no Exame deve constar em seu histórico escolar.

O Conceito Preliminar de Curso (CPC) foi criado pela Portaria Normativa MEC no 4 , de 5 de agosto de 2008, ratificado na Portaria ${ }^{\circ} 40 / 2007$, editada no ano de 2010, consolida os resultados do ENADE complementando o diagnóstico inicial traçado pelo indicador de Diferença entre os Desempenhos Observados e Esperado (IDD). Compreende alguns componentes de avaliação, como a infraestrutura e corpo docente da IES ${ }^{10}$.

O CPC é considerado oficialmente um indicador de Qualidade da Educação Superior do MEC e é constituído de acordo com o Quadro 1, são oito componentes, agrupados em três dimensões que têm por finalidade avaliar a qualidade dos cursos: o desempenho dos estudantes, o corpo docente e as condições para desenvolvimento do processo formativo ${ }^{12}$

Quadro 1. Composição do CPC e pesos das suas dimensões e composições.

\begin{tabular}{|c|c|c|c|}
\hline DIMENSÃO & COMPONENTES & \multicolumn{2}{|c|}{ PESOS } \\
\hline \multirow[b]{2}{*}{ Desempenho dos estudantes } & Nota dos concluintes no ENADE (ND) & $20,0 \%$ & \multirow[b]{2}{*}{$55,0 \%$} \\
\hline & $\begin{array}{l}\text { Nota do Indicador da Diferença entre os Desempenhos } \\
\text { Observados e Esperado (NIDD) }\end{array}$ & $35,0 \%$ & \\
\hline \multirow{3}{*}{ Corpo docente } & Nota de Proporção de Mestres (NM) & $7,5 \%$ & \multirow{3}{*}{$30,0 \%$} \\
\hline & Nota de Proporção de Doutores (ND) & $15,0 \%$ & \\
\hline & Nota de Regime de Trabalho (NR) & $7,5 \%$ & \\
\hline \multirow{3}{*}{$\begin{array}{l}\text { Percepção discente sobre } \\
\text { as condições do processo } \\
\text { formativo }\end{array}$} & Nota referente à organização didática- pedagógica (NO) & $7,5 \%$ & \multirow{3}{*}{$15,0 \%$} \\
\hline & Nota referente à infraestrutura e instalações físicas (NF) & $5,0 \%$ & \\
\hline & $\begin{array}{l}\text { Nota referente às oportunidades de ampliação da } \\
\text { formação acadêmica e profissional (NA) }\end{array}$ & $2,5 \%$ & \\
\hline
\end{tabular}

Fonte: INEP/DAES ${ }^{11}$.

O cálculo do CPC é realizado segundo a Nota Técnica n 3 de 2017/CGCQES/DAES ${ }^{11}$

A Nota Contínua do Conceito Preliminar de Curso (NCPC), calculada para cada curso de graduação, é uma variável contínua que pode assumir valores de 0 (zero) a 5 (cinco). A NCPC, convertida em faixa segundo os critérios expostos na Tabela 1, é transformada no Conceito Preliminar de Curso propriamente dito, que é uma variável discreta definida de 1 (um) a 5 (cinco). Os cursos de graduação com NCPC maior ou igual a 3,945 obterão o 
Tabela 1. Parâmetros de conversão da Nota Contínua do Conceito Preliminar de Curso em Conceito Preliminar de Curso.

\begin{tabular}{cc}
$\begin{array}{c}\text { CPC } \\
\text { (Faixa) }\end{array}$ & $\begin{array}{c}\text { NCPC } \\
\text { (Valor Contínuo) }\end{array}$ \\
\hline 1 & $0 \leq \mathrm{NC}<0,945$ \\
\hline 2 & $0,945 \leq \mathrm{NC}<1,945$ \\
\hline 3 & $1,945 \leq \mathrm{NC}<2,945$ \\
\hline 4 & $2,945 \leq \mathrm{NC}<3,945$ \\
\hline 5 & $3,945 \leq \mathrm{NC} \leq 5$ \\
\hline
\end{tabular}

Fonte: INEP/DAES ${ }^{11}$.

valor máximo do CPC (faixa igual a 5) somente se tiverem nota maior que 0,945 em todos os componentes. Caso contrário, mesmo obtendo NCPC maior ou igual a 3,945, o curso terá CPC igual a 4 (quatro). Essa nota é calculada para os cursos de graduação que possuam no mínimo 2 (dois) estudantes concluintes participantes no ENADE. Os cursos que não atendam a esse critério ficam na condição de "Sem Conceito" (SC).

Para a avaliação institucional, é atribuído o Índice Geral de Cursos Avaliados da Instituição (IGC), que também é um indicador de qualidade dos cursos das IES, e contempla a qualidade dos cursos de graduação e pós-graduação, conforme definição pelo INEP10:

O Índice Geral de Cursos Avaliados da Instituição (IGC) é um indicador utilizado para avaliar as Instituições de Ensino Superior, onde é calculado a média dos conceitos dos cursos de graduação (CPCs) em cada IES e de indicadores reativos aos programas de pós-graduação.

Neste estudo não foi utilizado como indicador de qualidade o IGC, devido ser o conceito geral referente às IES e não somente um indicador do curso de graduação.

Independente do perfil institucional de cada IES e das diferenças entre as cinco regiões brasileiras, os critérios de qualidade dos cursos de graduação e as Diretrizes Curriculares para os Cursos de Graduação são iguais para todos ${ }^{10}$.

\section{Método}

Foi realizado um estudo quantitativo, analítico e de caráter descritivo a partir de análise dos dados secundários coletados no Portal do Ministério da Educação, no Instituto Nacional de Estudos e Pesquisas, referentes às instituições de educação superior e cursos cadastrados autorizados para os cursos de graduação em fisioterapia através do portal eletrônico e-MEC, via busca interativa.

Foi realizado um levantamento em relação ao quantitativo das IES dos cursos de graduação em fisioterapia no Brasil cadastradas, por meio de consultas interativas ao sistema virtual do Ministério da Educação ${ }^{13}$ nos meses de abril a setembro de 2016 em duas fases.

Na primeira fase, foi coletado os seguintes dados: nome da instituição; nome da mantenedora; categoria administrativa das IES; organização acadêmica das IES; endereço; CEP; bairro; telefone; município, estado; Conceito Preliminar do Curso (CPC), este com avaliação até o ano de 2013 (notas do último ENADE publicado). As IES sem conceito são as que não tinham egressos ou ainda não haviam aderido ao Sistema Nacional de Avaliação (SINAES), no caso das IES estaduais.

Ressaltando que, neste estudo não foram contabilizados os cursos na modalidade EAD cadastradas no sistema e-MEC.

Na segunda fase foi gerado um Banco de Dados Geográfico (BDGEO) por meio da indexação das coordenadas geográficas $\mathrm{X}$ e $\mathrm{Y}$ de cada localização das IES que possuem cursos de fisioterapia no Brasil. A partir de então, foi consultado os códigos dos municípios e estados através do banco de dados do IBGE de cada IES coletada. Posteriormente foram 
gerados mapas temáticos com a distribuição espacial das IES de fisioterapia por regiões e unidades federativas (UF). Para produzir os mapas foi utilizado o software ARCGIS 10.2.

Por se tratar de análise documentais, esta pesquisa foi dispensada da análise pelo Comitê de Ética em Pesquisa Envolvendo Seres Humanos do CCBS- UEPA, pelo Protocolo 05/16 de 25 de abril de 2016. Esta pesquisa é parte da dissertação de mestrado em Ensino Em Saúde na Amazônia da Universidade do Estado do Pará.

\section{Resultados}

Atualmente foram identificadas 632 IES com o curso de fisioterapia na modalidade de ensino presencial. Estas IES estão distribuídas pelos estados brasileiros conforme apresentado na Tabela 2.

Ao analisarmos o caráter administrativo, público ou privado das 632 IES distribuídas por região, verificamos que a maioria dos cursos avaliados concentram -se na Região Sudeste, sendo $28,21 \%$ são de IES públicas e $44,08 \%$ são IES privadas, respectivamente (Tabela 3).

Tabela 2. Quantidade de IES cadastradas no sistema e-MEC por UF e DF³.

\begin{tabular}{|c|c|c|}
\hline UF e DF & Quantidade de IES & $\%$ \\
\hline Acre & 3 & 0.47 \\
\hline Alagoas & 12 & 1.90 \\
\hline Amapá & 4 & 0.63 \\
\hline Amazonas & 8 & 1.27 \\
\hline Bahia & 51 & 8.07 \\
\hline Ceará & 19 & 3.01 \\
\hline Distrito Federal & 13 & 2.06 \\
\hline Espírito Santo & 13 & 2.06 \\
\hline Goiás & 25 & 3.96 \\
\hline Maranhão & 8 & 1.27 \\
\hline Mato Grosso & 15 & 2.37 \\
\hline Mato Grosso do Sul & 10 & 1.58 \\
\hline Minas Gerais & 68 & 10.76 \\
\hline Pará & 11 & 1.74 \\
\hline Paraíba & 15 & 2.37 \\
\hline Paraná & 38 & 6.01 \\
\hline Pernambuco & 27 & 4.27 \\
\hline Piauí & 15 & 2.37 \\
\hline Rio de Janeiro & 35 & 5.54 \\
\hline Rio Grande do Norte & 10 & 1.58 \\
\hline Rio Grande do Sul & 38 & 6.01 \\
\hline Rondônia & 10 & 1.58 \\
\hline Roraima & 2 & 0.32 \\
\hline Santa Catarina & 26 & 4.11 \\
\hline São Paulo & 143 & 22.63 \\
\hline Sergipe & 7 & 1.11 \\
\hline Tocantins & 6 & 0.95 \\
\hline Total & 632 & 100.00 \\
\hline
\end{tabular}

Fonte: Dados da pesquisa, 2017. 
Tabela 3. Quantidade e Percentual por Categoria Administrativa das Instituições de Ensino Superior com Conceito Preliminar de Curso por Região.

\begin{tabular}{cccccc} 
& \multicolumn{3}{c}{ Privada } & & \multicolumn{2}{c}{ Público } \\
\cline { 2 - 3 } \cline { 5 - 6 } Região & Quantidade & Percentual & & Quantidade & Percentual \\
\cline { 2 - 3 } Centro-Oeste & 33 & 10,86 & & 04 & 10,26 \\
\hline Nordeste & 66 & 21,71 & & 11 & 28,21 \\
\hline Norte & 20 & 6,58 & & 03 & 7,69 \\
\hline Sudeste & 134 & 44,08 & & 11 & 28,21 \\
\hline Sul & 51 & 16,78 & & 10 & 25,64 \\
\hline Total & $\mathbf{3 0 4}$ & $\mathbf{1 0 0 , 0 0}$ & & $\mathbf{3 9}$ & $\mathbf{1 0 0 , 0 0}$ \\
\hline
\end{tabular}

Fonte: Dados da pesquisa, 2017.

O mapa da distribuição pontual das Instituições de Ensino Superior dos Cursos de fisioterapia no Brasil apresenta a localização exata através das coordenadas geográficas, podemos evidenciar uma sobreposição de IES, pelo fato de estarem concentradas em grande parte nas capitais e regiões metropolitanas (Figura 1).

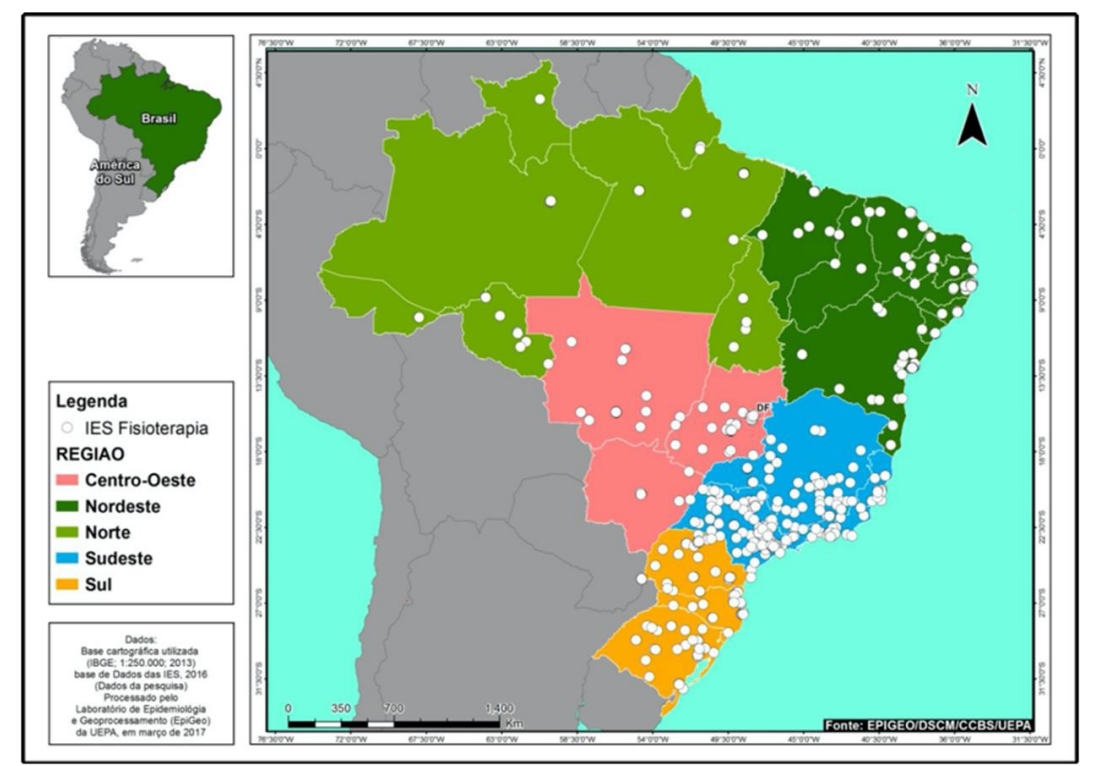

Figura 1. Distribuição pontual das Instituições de Ensino Superior dos cursos de fisioterapia no Brasil. Fonte: Dados da pesquisa, 2017.

Ao compararmos as regiões brasileiras, a maioria dos cursos que foram avaliados e que apresentaram CPC estão localizados na região Sudeste, enquanto que a região Norte apresenta o menor número, denotando uma significativa diferença (Figura 2).

A Figura 2 apresenta o quantitativo e o percentual das IES por região com e sem Conceito Preliminar de Curso. Os cursos de fisioterapia sem conceito (SC) são aqueles que não apresentaram o Conceito ENADE/ Conceito Preliminar de Curso (CPC) pelas seguintes razões: podem ser cursos iniciantes (novos) que ainda não têm alunos concluintes; podem também ser cursos que não obtiveram a nota mínima do conceito, ou ainda podem aqueles que não atualizaram as informações no banco de dados do Portal e-MEC, no período da busca interativa.

A Tabela 4 apresenta a quantidade de IES por região com Conceito Preliminar do Curso (CPC) por faixa.

O cálculo do CPC é realizado segundo a Nota Técnica n 3 de 2017/CGCQES/DAES ${ }^{11}$, é uma variável contínua que pode assumir valores de 0 (zero) a 5 (cinco) onde zero é a menor nota (faixa) e cinco a maior nota (faixa). 


\section{ighe}

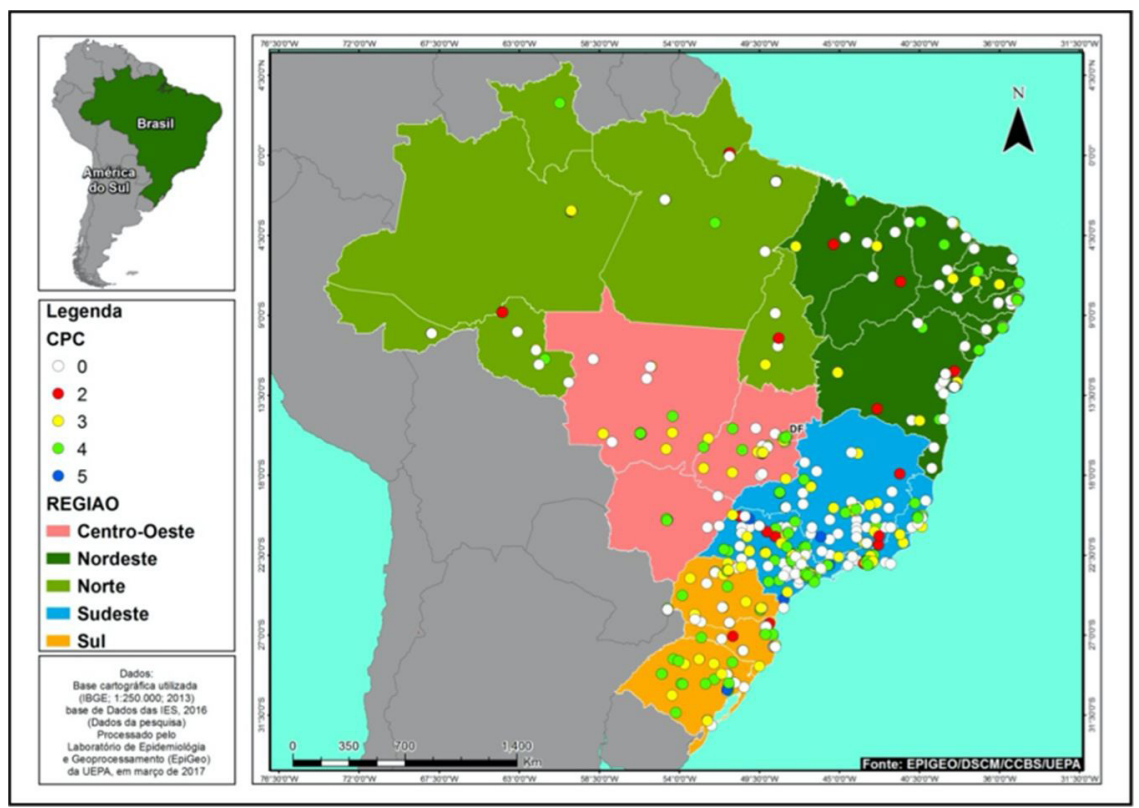

Figura 2. Distribuição pontual do Conceito Preliminar de Curso para os cursos de graduação em fisioterapia no Brasil.

Fonte: Dados da pesquisa, 2017.

Tabela 4. Quantidade de Instituição de Ensino Superior por região com Conceito Preliminar de Curso por Faixa.

\begin{tabular}{lcccc} 
& \multicolumn{5}{c}{ CPC POR FAIXA } \\
\cline { 2 - 5 } \multicolumn{1}{c}{ Região } & 2 & 3 & 4 & 5 \\
\hline Centro-Oeste & 3 & 17 & 17 & 0 \\
\hline Nordeste & 7 & 44 & 5 & 0 \\
\hline Norte & 7 & 11 & 61 & 0 \\
\hline Sudeste & 12 & 64 & 33 & 3 \\
\hline Sul & 2 & 23 & 36
\end{tabular}

Fonte: Dados da pesquisa, 2017.

Observa-se que na Região Sudeste há uma maior concentração de cursos, totalizando 259. Destes, 145 tem CPC atribuído, distribuídos da seguinte forma: 12 apresentam conceito insatisfatório, 125 apresentam conceito na faixa 3 e 4 e 8 cursos na faixa 5.

Já as Regiões Norte e Nordeste apresentaram respectivamente 7 IES com conceito insatisfatório e a maioria do CPC dos cursos estão distribuídos nas faixas 3 e 4 e nenhum na faixa 5.

As regiões Sul e Sudeste são as únicas que apresentaram cursos com conceito máximo.

\section{Discussão}

A região Norte, composta pelos estados do Acre, Amazonas, Rondônia, Roraima, Amapá, Pará e Tocantins, possui 8,59\% da população do Brasil onde se localizam 6,95\% das IES com cursos graduação em Fisioterapia.

Embora muitos dos indicadores epidemiológicos e sociodemográficos tradicionais tenham apresentado no país melhoras nos últimos vinte anos no Norte, os avanços têm sido lentos e irregularmente distribuídos, privilegiando os principais centros urbanos da região em detrimento do grande contingente populacional das zonas rurais. 
Os estados de Alagoas, Bahia, Ceará, Maranhão, Paraíba, Pernambuco, Piauí, Rio Grande do Norte e Sergipe formam a região Nordeste e nela se encontram 27,62\% da população brasileira e $26,07 \%$ dos cursos de graduação em Fisioterapia.

O Nordeste é a terceira maior região do Brasil, apesar do Produto Interno Bruto (PIB) representar apenas 13\%. Embora as políticas de desenvolvimento e de redução das desigualdades estejam presentes, a região ainda apresenta grande desigualdade socioeconômica.

Composta pelos estados de Goiás, Mato Grosso, Mato Grosso do Sul e Distrito Federal, a região Centro-Oeste possui 7,60\% da população do Brasil e 9,95\% das IES com o curso de Fisioterapia.

O Centro-Oeste ocupa a penúltima posição referente ao número de IES no país, na frente apenas da região Norte. De todas as regiões brasileiras, é a que compõe a mais alta taxa de escolarização líquida para o ensino superior. Também é a região que apresenta a maior proporção de IES privadas, com projeção crescente para a área da saúde ${ }^{14}$.

Na região Sudeste, formada pelos estados do Espírito Santo, Minas Gerais, Rio de Janeiro e São Paulo, estão localizados 40,92\% dos cursos de graduação em Fisioterapia e $41,90 \%$ da população brasileira, correspondendo à maior porcentagem no quantitativo populacional e de IES ${ }^{14}$

No Sudeste está a maior concentração de IES e o maior número de cursos e estudantes dentre as cinco regiões, apresentando alta qualificação docente e a maior proporção de doutores do país ${ }^{14}$

Com $14,29 \%$ da população do Brasil, a região Sul é constituída pelos estados do Paraná, Santa Catarina e Rio Grande do Sul, onde se localiza 16,11\% das IES com cursos de Fisioterapia.

A região Sul é a que apresenta maior quantitativo de IES fora das capitais. Com o setor industrial fixado nos interiores, houve um aumento da demanda por mão de obra qualificada, o que gerou novas IES ${ }^{14}$.

Nas regiões onde há maior desenvolvimento econômico, observou que ocorre uma maior concentração de IES e um número maior de profissionais disponíveis para o mercado de trabalho e um maior percentual de IES de categoria administrativa privada, caracterizando a influência do desenvolvimento econômico nestas variáveis ${ }^{15}$.

Os resultados encontrados demonstraram uma desigualdade na distribuição dos cursos entre as regiões, havendo também uma grande concentração nas cidades de grande porte e de maior densidade populacional.

Outras razões que podem justificar as desigualdades regionais são as altas concentrações de recursos e tecnologias em algumas regiões, principalmente nas capitais, em oposição à escassez de profissionais, tecnologias e capacidade de investimento, agregada a diversidade socioespacial de alguns territórios, a exemplo da região Norte $^{16}$.

Com a criação de indicadores para a avaliação da qualidade do ensino superior expressos por faixa numérica (conceito), leva alguns autores a fazer críticas a respeito da sua utilização, por gerarem uma exposição na mídia como um ranking, favorecendo o uso do marketing institucional a fim de promover a IES, embora o seu real significado esteja "escondido" na propaganda das IES".

Enquanto outros autores como Barreyro e Rothen ${ }^{17}$ alegam que comparações e resultados não são negativos, podendo funcionar como um fator de concorrência à demanda do mercado, bem como a colaboração e melhoria da qualidade do ensino.

A análise dos resultados publicados nos relatórios do ENADE (2008), refletidos nos Conceitos Preliminares dos Cursos, serviram de base para sensibilização dos alunos e dos docentes em relação ao desempenho dos estudantes.

Como consequência, foram apresentadas algumas propostas que viessem a contribuir com um melhor resultado no conceito dos cursos, sendo esta uma das sugestões apresentadas: utilização de métodos e técnicas para elaborar questões que 
avaliem os conhecimentos, as habilidades e competências preconizados nas DCN e nos projetos pedagógicos, com a adequação da matriz curricular a realidade, afim de que os gestores pudessem ter subsídios para correções no percurso do processo de ensino-aprendizagem do aluno.

Dessa forma, as avaliações no cotidiano dos cursos deveriam ser embasadas nos mesmos princípios e contextos que o Exame Nacional de Desempenho do Estudante atua, sendo esta uma das principais contribuições deste exame para melhoria da qualidade do ensino superior no Brasil ${ }^{18}$.

\section{Conclusão}

O presente estudo permitiu caracterizar a distribuição e a qualidade dos cursos de graduação em fisioterapia no Brasil por regiões e estados.

É importante que as Instituições de Ensino Superior reavaliem os critérios referentes à qualidade dos cursos de Fisioterapia. Para tal, faz-se necessário o envolvimento no ensino-aprendizagem de forma coesa, eficiente e eficaz tendo por base o projeto pedagógico do curso com uma matriz curricular capaz de traduzir os critérios essenciais para a formação profissional de acordo com as diretrizes curriculares de graduação em Fisioterapia, e com as necessidades da população nos aspectos socioeconômicos e epidemiológicos.

Espera-se contribuir com o planejamento de abertura de novos cursos nas regiões estrategicamente apresentadas, possibilitando a implementação de políticas de qualificação no ensino superior em Fisioterapia e a integração ao Sistema Único de Saúde para os interiores dos estados, com programas que possam incluir o profissional diretamente na assistência à saúde.

\section{Referências}

1. Barros FMB. Poliomielite, filantropia e fisioterapia: o nascimento da profissão de fisioterapia no Rio de Janeiro dos anos 1950. Cien Saude Colet. 2008;13(3):941-54. http://dx.doi.org/10.1590/S1413-81232008000300016. PMid:18813587.

2. Bispo JPJ. Formação em Fisioterapia no Brasil: reflexões sobre modelos de formação. Hist Cienc Saude Manguinhos. 2009;16(3):655-68. http://dx.doi.org/10.1590/S0104-59702009000300005. PMid:20614669.

3. Rebelatto JR, Botomé SP. Fisioterapia no Brasil. Fundamentos para uma ação preventiva e perspectivas profissionais. 2. ed. São Paulo: Manole.

4. Fernandes JD, Teixeira GAS, Silva MG, Florêncio RMS, Silva RMO, Rosa DOS. Expansão da educação superior no Brasil: ampliação dos cursos de graduação em enfermagem. Rev Latino-Am Enfermagem, 2013 Maio-Jun;21(3):1-8.

5. Canopf L, Festinalli RC, Ichikawa EY. A expansão do ensino superior em administração no sudoeste do Paraná: reflexões introdutórias. Rev Adm Contemp. 2005 Jul-Set;9(3):79-97.

6. Ribeiro MA, Albuquerque IMN, Paiva GM, Vasconcelos JPC, Araújo MAVF, Vasconcelos MIO. Georreferenciamento: ferramenta de análise do sistema de saúde de Sobral - Ceará. SANARE. 2014;13(2):63-9.

7. Kuschnir R, Chorny AH. Redes de atenção à saúde: contextualizando o debate. Cien Saude Colet. 2010;15(5):2307-16. http://dx.doi.org/10.1590/S1413-81232010000500006. PMid:20802864.

8. OMS: Organização Mundial da Saúde. Increasing access to health workers in remote and rural areas through improved retention: global policy recommendations. Genebra: OMS; 2010.

9. Macedo AR, Trevisan LMV, Trevisan P, Macedo CS. Educação superior no século XXI e a reforma universitária brasileira. Ensaio: Aval Pol Públ Educ. 2005;13(47):127-48. http://dx.doi.org/10.1590/S0104-40362005000200002.

10. INEP: Instituto Nacional de Estudos e Pesquisas Educacionais Anísio Teixeira [Internet]. Brasília: INEP; 2015 [citado em 2016 Dez 15]. Disponível em: http://www.inep.gov.br

11. INEP: Instituto Nacional de Estudos e Pesquisas Educacionais Anísio Teixeira [Internet]. Brasília: INEP; 2017 [citado em 2017 Mar 2]. Disponível em: http://www.inep.gov.br

12. Ikuta CYS. Sobre o conceito preliminar de curso: concepção, aplicações e mudanças metodológicas. Est Aval Educ. 2016;27(66):938-69. http://dx.doi.org/10.18222/eae.v27i66.4039.

13. Brasil. Ministério da Educação. E-MEC: Instituições de Educação Superior e cursos cadastrados [Internet]. Brasília: Ministério da Educação; 2017 [citado em 2017 Mar 2]. Disponível em: www.emec.gov.br

14. GE Ensino Superior. Guia do Estudante. São Paulo: Editora Abril; 2016.

15. Tavares LRC, Costa JLR, Oishi J, Driusso P. Distribuição territorial de fisioterapeutas no Brasil: análise do Cadastro Nacional de Estabelecimentos de Saúde CNES/2010. ConScientiae Saúde. 2016;15(1):53-61. http://dx.doi.org/10.5585/conssaude. v15n1.6152. 


\section{ighe}

16. Lima LD, Viana ALÁ, Machado CV, et al. Regionalização e acesso à saúde nos estados brasileiros: condicionantes históricos e político - institucionais. Cien Saude Colet. 2012;17(11):2881-92. http://dx.doi.org/10.1590/S1413-81232012001100005. PMid:23175295.

17. BARREYRO, G.B.; ROTHEN, J.C. Percurso da avaliação da educação superior nos Governos Lula. Educ. Pesqui., São Paulo, v. 40, n.1, p. 61-76, jan./mar, 2014.

18. Castro LSF. Desenvolvimento de um método de gestão do ambiente de ensino aprendizagem em moléstias infecciosas aplicadas ao curso de medicina no Pará [tese]. Belém (PA): Programa de Pós-graduação Núcleo de Medicina Tropical, Universidade do Estado do Pará; 2014. 129 p.

\section{Contribuição dos autores}

Erica Silva de Souza Matsumura participou da Concepção e desenvolvimento da pesquisa, da confecção do desenho metodológico, da coleta e tratamento dos dados e da Análise, interpretação dos resultados. Alcinês da Silva Sousa Júnior realizou a supervisão da pesquisa, a redação do artigo, e a análise, interpretação. Nelson Veiga Gonçalves participou do elaboração do desenho metodológico, da análise, interpretação dos resultados e da revisão bibliográfica. Katia Simone Kietzer participou da Concepção e desenvolvimento da pesquisa, da supervisão do projeto e do manuscrito e da revisão crítica do manuscrito. Leny Silene de Freitas Castro participou da Concepção e desenvolvimento da pesquisa, da supervisão do projeto e do manuscrito e da revisão crítica do manuscrito. 